\title{
Solving challenging bioorganic problems by exciton coupled CD
}

\author{
Nina Berova, Babak Borhan, Jian Guo Dong, Jinsong Guo, Xuefei Huang, Elena Karnaukhova, \\ Akira Kawamura, Jihong Lou, Stefan Matile, Koji Nakanishi, Barry Rickman, Jing Su, Qiang \\ Tan, and Irini Zanze
}

Department of Chemistry, Columbia University, New York, NY 10027, USA

Abstract - Determination of molecular chirality is becoming increasingly important due to the sophistication in mode of action studies of biological reactions and the importance of chiral drugs. The exciton coupled $C D$, based on through space coupling, is a versatile microscale method with superb sensitivity that can be applied to a variety of problems including biopolymers and clusters in lipids. Applications of the exciton coupled CD method to the following problems exemplify its versatility.

\section{Quaternary ammonium salts as chromophores for exciton-coupled CD. ${ }^{1 *}$}

Exciton coupled circular dichroism is a nonempirical submicrogram scale method for determining absolute configurations and conformations of organic molecules in solution based on the interactions of chromophores which are preexisting in the substrate and/or introduced through derivatizations such as $\mathrm{O}$ - or $\mathrm{N}$-acylation. 2,3 However, the method could not be applied to one major group, i.e., tertiary amines which comprise numerous natural products, particularly alkaloids, and synthetic drugs that exhibit conspicuous biological activities. We have succeeded in extending the $C D$ method to quinuclidines (tertiary amines) through quaternary ammonium salt formation, and have applied this new procedure to the important class of hypocholesterolemic

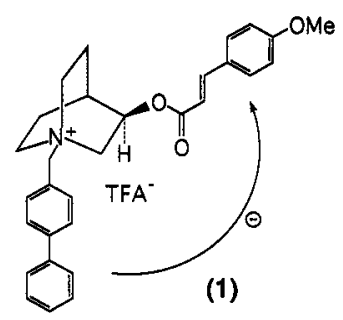

(1) quinuclidines. Of the three conformers conceivable for the salt (1), the conformer shown determines the sign of the split Cotton effect (CE). When the phenylbenzyl group is in the north east sector the transition moments of the two chromophores are close to being parallel, hence the chirality is negligible; when it is in the north west sector, the distance between the two chromophores is more remote than that depicted, hence the resulting positive bisignate $C D$ will be of smaller amplitude.

\section{Acyclic 1,3-polyols with a 1,2-diol terminal. 4}

Acyclic 1,3-polyols are widely distributed in nature. Particularly 1,2,4-triols are present in numerous antifungal polyene macrolides in various masked forms. Although over 200 polyene macrolides are known, the structures of only about 40 have been determined, while those for which the full stereochemistry has been elucidated are less than ten. In order to develop a general chiroptical method for structure determination of acyclic 1,3-polyols, we have combined a divergent synthetic approach with $C D$ to prepare all possible stereoisomers of 1,2,4-triols, 1,2,4,6tetrols and 1,2,4,6,8-pentols. The current set of reference polyols should be useful for setting up reference $C D$ libraries and for model studies leading to a general method for configurational assignment of acyclic polyols. CD curves of 1,2-diols, 1,2,4-triols, and 1,2,4,6-tetrols with differing stereochemistries have been published earlier. ${ }^{5}$

\section{Synthesis of cyclic mixed polyols ${ }^{6}$ and acyclic pentol structure of two hopanoids. ${ }^{7}$}

The synthesis of all eight configurational isomers of acyclic 1,2,3,4,6-pentols belonging to the $2 R$ enantiomeric series has been carried out, and the side-chain configurations of two bacteriohopanoids (2) and (3) have been established by microgram scale derivatization and $C D$. 


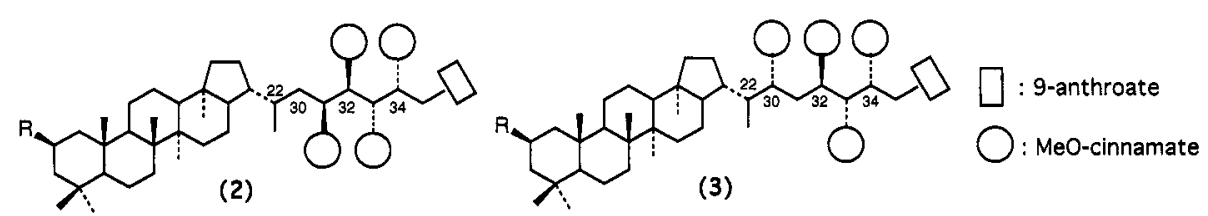

\section{Calculations of vinblastine alkaloid $\mathrm{CD} .^{8}$}

SCF-CI-dipole velocity $\mathrm{MO}$ calculations have shown that the diagnostically useful bisignate $C D$ curves of vinblastine $(R=M e) /$ vincristine $(R=C H O)$ alkaloids at ca. $210 \mathrm{~nm}$ and 220 $230 \mathrm{~nm}$ are due to exciton coupling between the indoline and indole moieties. Furthermore, a combination of X-ray crystal structure data with MM2 local energy minimization provides a convenient means for estimation of the preferred solution conformation.

\section{Search for endogenous mammalian cardiotonic factor. $9 \mathrm{a}$}

The specific, high affinity binding of plant-derived digitalis glycosides by the mammalian sodium and potassium transporting adenosine triphosphatase ( $\mathrm{Na}, \mathrm{K}$-ATPase, or sodium pump), a plasma membrane enzyme with critical physiological importance in mammalian tissues, has raised the possibility that a mammalian analog of digitalis might exist. We previously isolated and structurally characterized from bovine hypothalamus a novel isomer of the plant

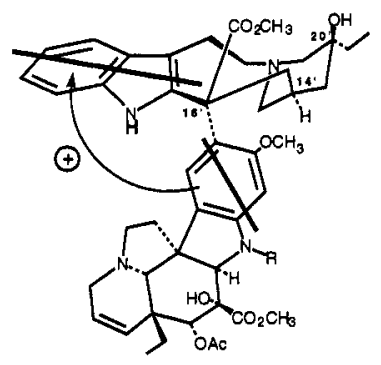
glycoside, ouabain, which differs structurally only in the attachment site and/or the stereochemistry of the steroid moiety. $9 \mathrm{~b}$ Hamlyn and co-workers reported a molecule purified from human plasma which by mass spectrometry could not be distinguished from plant ouabain. $9 \mathrm{c}$ Since rhamnoside cardiotonic steroids are not known as natural products from mammalian sources, it became important to compare these two pure isolates to determine if the same or structurally distinct compounds had been found. The factors from bovine and human, $300 \mathrm{ng}$ each, were converted into their pentanaphthoates by sub-microgram manipulations and their HPLC and CD

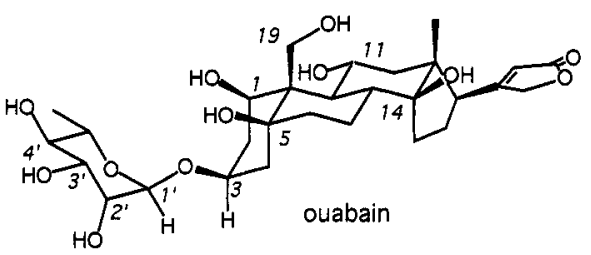
were compared with the corresponding derivative from ouabain. The two mammalian factors were identical both exhibiting almost nil CD (!), whereas the ouabain pentanaphthoate showed a strongly positively coupled split $C D, A=+472$ centered at $233 \mathrm{~nm}$ (in $\mathrm{MeCN}$ ). Thus the human and bovine $\mathrm{Na}, \mathrm{K}-$ ATPase-inhibitors are identical, but different from plant ouabain. This supports the notion that the human sodium pump may be under specific physiological regulation by a mammalian analog of the digitalis glycosides. ${ }^{9}$

\section{Induced chirality with auxiliary chiral moieties. ${ }^{10 a}$}

There are cases in which exciton coupling between two chromophores does not occur because the two electric transition moments which should interact are coplanar. Such are the cases in cyclohexane-1,4-diols (both ee or ea), and a wide variety of 3-hydroxycarotenoids, 3hydroxyretinoids, etc. A general approach to cope with such cases is to acylate one of the hydroxyl groups with a chiral allenic acid substituted with a suitable chromophore, e.g., CHROM$\mathrm{CH}=\mathrm{C}=\mathrm{CH}-\mathrm{COOH}$. The allenic bond introduces a $90^{\circ}$ twist at the central allenic carbon so that the allenic CHROM now couples with the second chromophore.10a This concept of introducing an auxiliary allenic center should have general applicabilities in other similar cases. However, since the allenic ester was found to be too reactive to be of general use, other auxiliary chiral molecules are under investigation. $10 \mathrm{~b}$ 


\section{Sphingosine analysis - 1 microgram scale. ${ }^{11}$}

Breakdown products of sphingolipids are involved in cell regulation and exhibit a wide variety of activities related to signal transduction. The backbone of sphingolipids is composed of the sphingosines, which have two stereogenic centers. A general, rapid, chemical/chiroptical micromethod based on the bichromophoric CD exciton chirality principle has been developed to distinguish all four possible stereoisomers nonambiguously at a level of ca. 1 microgram. The protocol is based on the bichromophoric exciton coupled $C D$ method, a very sensitive, nonempirical microscale approach for determining the absolute configuration of cyclic or acyclic compounds

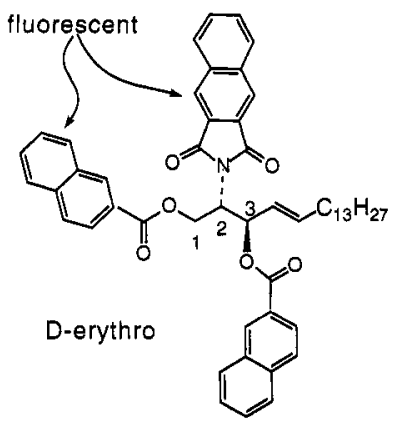
in solution. The employment of two different strongly absorbing chromophores leads to characteristic exciton split CD curves which depend on the absolute skewness of interacting chromophores and pairwise additivity principle. In order to obtain diagnostic $C D$ reference curves for all four sphingosine diastereomers, $D / L$ erythro and $\mathrm{D} / \mathrm{L}$ threo, numerous bichromophoric combinations, including new chromophores, were tested over the years. Two basic approaches were envisaged: (i) selective derivatization of the primary hydroxyl group with a bulky chromophore, followed by $O, N$-acylations with a second chromophore, including formation of Schiff bases from the primary amino group, (ii) $O$ derivatization and $\mathrm{N}$-derivatization with different chromophores. However, these routes also had to be abandoned because the derivatizations were not suited at the microscale and/or Schiff bases were not sufficiently stable for performing HPLC separations.

The multiple problems were finally solved by converting the amino group into the fluorescent and stable naphthimido chromophore, and then converting the $1^{\circ}$ - and $2^{\circ}$ - hydroxyls into 2-naphthoate esters, also fluorescent. The fluorescence allows the reactions to be performed at submicrogram levels.

\section{Sphingosines and dihydrosphingosines. 12}

The above-mentioned method has been further improved and developed into a simple picomole (low nanogram) scale HPLC scheme. This protocol can separate all eight sphingosines and dihydrosphingosines thus leading to the identification of their relative and absolute configurations. The amino group of the sample is derivatized to its fluorescent $N$-naphthimide which is analyzed by normal and chiral phase HPLC, coupled with fluorescence peak detection. If necessary, the results of this HPLC method can be further corroborated by measurements of CD spectra of the $\mathrm{N}$-naphthimido-derivatives and/or $\mathrm{N}, \mathrm{O}$-chromophoric derivatives.

\section{Porphyrins as extremely powerful chromophores for exciton coupled CD.13,14}

In exciton coupled $C D$, in most cases, acylating chromophores are introduced into the substrate, and the sign of split CD resulting from the coupling between the chromophores are used to determine the absolute sense of twist. This is a very versatile method but the extent of its potentiality has yet to be explored, particularly in conformational studies of large molecules exemplified by biopolymers. The extent of exciton coupling is inversely proportional to the square of interchromophoric distance and proportional to the square of extinction coefficients of the coupled chromophores. The latter properties of exciton coupled CD could hinder studies with large biopolymers. Therefore, the intensity of chromophoric absorptions is of prime importance in increasing the sensitivity over the large distance between interacting transition moments. Thus, in large molecules such as receptors, proteins, nucleic acids, etc., the availability of chromophores with intense absorptions in a region that does not overlap with the substrate absorption is of prime importance. To date no such chromophores are known or have been used in exciton coupled CD.

Porphyrins, e.g., 5-p-carboxyphenyl-10,15,20-triphenylporphyrin, characterized by a sharp Soret band at $414 \mathrm{~nm}, \varepsilon=350000$, has proven to be promising new exciton coupled CD chromophores. It readily acylates both hydroxyl and amino groups, produces a couplet at ca. 415 $\mathrm{nm}$, and enhances the sensitivity over $\mathrm{p}$-dimethylaminobenzoate (or benzamide), one of the most sensitive chromophores, by 7 to 9 -fold. 13 

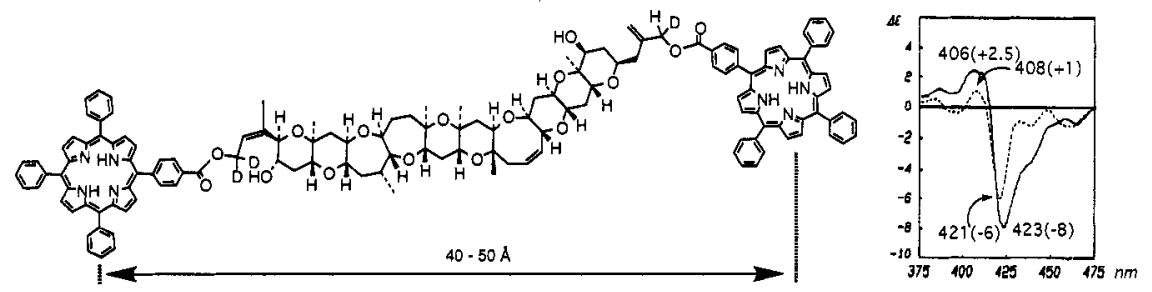

Structure and CD of brevetoxin-bridged porphyrin dimer in $\mathrm{MeOH} /$ water $4 / 1$ in the presence (solid) and absence (dotted) of $\mathrm{CsCl}, \mathrm{C}=1.0 \mu \mathrm{M}$.

Further studies ${ }^{14}$ have delineated the scope and limitations of porphyrin chromophores for structural studies by the exciton coupled CD method. The direction of the electric transition moment of the porphyrin chromophore has been determined to run in the 5-15 direction or in the direction of the $\mathrm{C}-\mathrm{O}$ or $\mathrm{C}-\mathrm{N}$ bond connecting the $\mathrm{OH}$ or amino groups to the porphyrin chromophore (as in the case of other acylates). A distance dependency of the porphyrin coupling was investigated in the range between $10 \AA$ to $50 \AA$ (e.g., brevetoxin derivative). Over short interchromophoric distances, significant changes in the conformational distribution introduced by the bulky porphyrin chromophores were observed. Over longer distances, the porphyrins showed ca.10-fold sensitivity increase over commonly used chromophores, and an effective direction for the interacting porphyrin transition moments was assigned by comparison. Porphyrins at the termini of dimeric steroids and brevetoxin $B$ exhibited exciton coupling over interchromophoric distances up to $50 \AA$. These results represent the porphyrins as promising reporter chromophores for extending the exciton coupled $C D$ method to structural studies of biopolymers.

10. Absolute configurational assignment of acyclic compounds with single chiral centers. ${ }^{15}$

We report a new concept based on exciton coupled circular dichroism (CD) for assigning absolute configurations to a single chiral center ${ }^{*} \mathrm{CXYSL}$, where $\mathrm{X}$ is $-\mathrm{OH}$ or $-\mathrm{NH}_{2}, \mathrm{Y}$ is an acyclic chain with terminal $\mathrm{OH}$ or $-\mathrm{NH}_{2}$, and $\mathrm{S}$ (small) and $\mathrm{L}$ (large) represent sterically distinct groups.
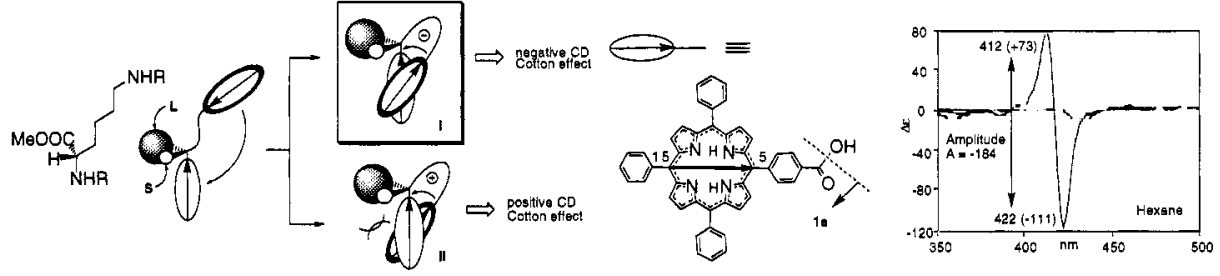

It consists of a one step attachment of porphyrins to $X$ and $Y$ followed by CD measurement. The key event is intramolecular porphyrin $\pi, \pi$-stacking, which converts the flexible, acyclic substrate into a rigid stacked conformation characterized by bisignate exciton split $C D$ curves. Since the stacked conformer is sterically controlled by the two groups, $\mathrm{S}$ and L, the sign of the exciton split $\mathrm{CD}$ is directly governed by the spatial arrangement of these groups. This approach is applicable to various acyclic compounds with different $C / C$ distances $(1,3 \sim 1,15)$ between the functional groups, but not to $1,2-\mathrm{C} / \mathrm{C}$. L-lysine bisporphyrin shows the expected strong negatively split couplet.

\section{Self-assembled brevetoxin-porphyrin conjugates. ${ }^{16}$}

Brevetoxins, involved in the "red tide" as well as shellfish poisoning, are known to bind to cell membranes and membrane proteins. Brevetoxin $B$ (BTX-B) interacts specifically with neuronal sodium channels. However, we recently found that BTX also induces selective ion movements across lipid bilayers through transmembrane BTX self-assemblies. 17 The supramolecular chemistry involved in the interaction of BTX with itself, cations, and lipid bilayers has been studied using the powerful porphyrin chromophores as $\mathrm{CD}$-labels. The structure of the active BTX-B complex in

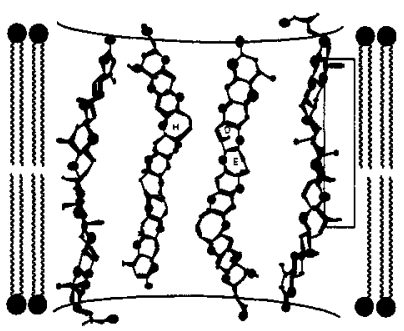


lipid bilayers is a cyclic, transmembrane self-assembly consisting of antiparallel aligned BTX molecules; similar ionophoric BTX self-assemblies have also been characterized in $\mathrm{MeOH} /$ water. In the Figure shown it is arbitrarily assumed that ca. six molecules form a cylinder (two in the front are not depicted). Structural studies have shown that the critical "micelle" concentrations (cmc) of different BTXs dictate specific mechanisms of pore formation.

\section{Second derivative $C D$ of ligand/receptor adducts. 18}

In the case of rapamycin/FKBP (FK 506 binding protein) adducts, a comparison of the second derivative adduct $C D$ with the rapamycin and FKBP summation CD disclosed very subtle conformational changes occurring with bioactive rapamycins upon complexing with FKBP; this difference was not observed with inactive rapamycins. This subtle change which involves a stiffening and flattening of the triene moiety of active rapamycins cannot be detected by NMR nor by X-ray of the adduct. The techniques of second derivative $C D$ and difference $C D$ promise to be a further powerful tool in dealing with complex $C D$, especially those involving

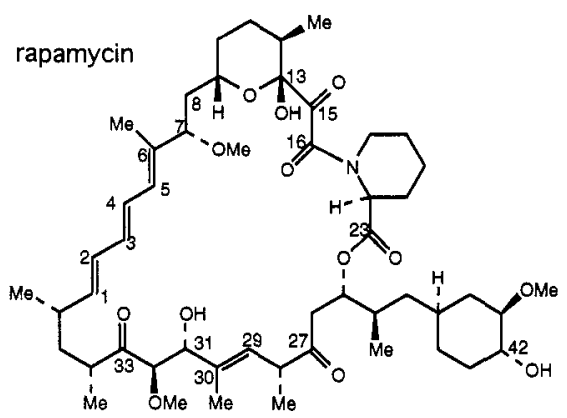
biopolymers.

13. Absolute sense of twist of the retinal chromophore in rhodopsin.19, 20

Many years were spent in attempts to perform photoaffinity labeling with rhodopsin in order to define the location of the retinal chromophore within the binding site. Clearcut results were finally secured by employing tritiated 3-diazo-4-keto retinal having an 11-cis-locked side chain which blocks the cis $\rightarrow$ trans isomerization during photoactivation (Fig. A). ${ }^{19}$ The results

intradiscal side

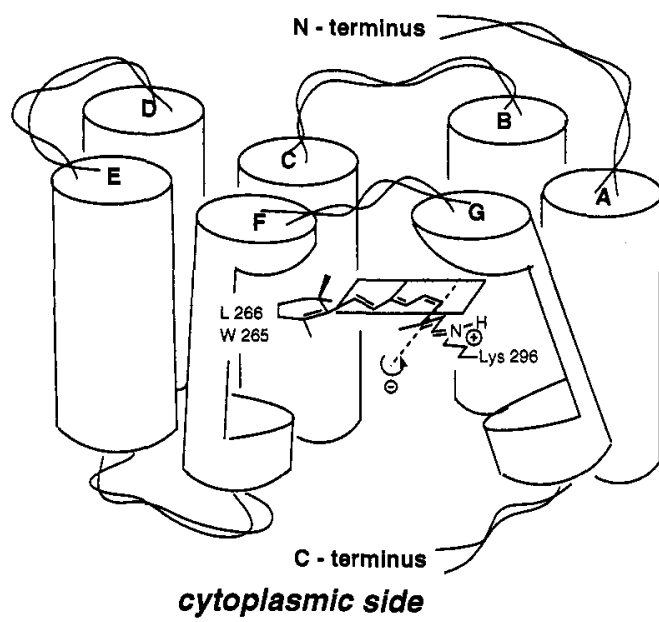

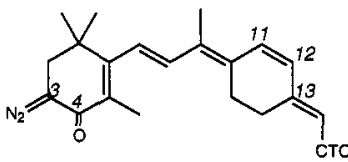

Zhang et al., J.Am.Chem.Soc.,116, 10165 ('94) Nakanishi et al., Biophys. J.56, 13 ('95)

Fig. A

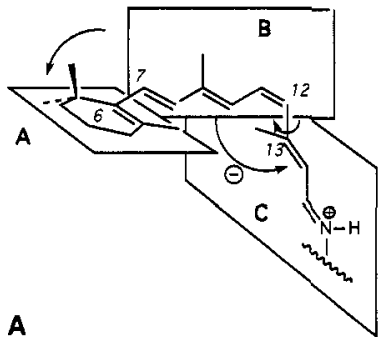

showed that the carbene generated at $\mathrm{C}-3$ had cleanly cross-linked to two amino acid residues, Trp-265 and Leu-266, i.e., the ionone C-3/C-4 was close to helix $F$ of the seven transmembrane alpha-helices (Fig. A). ${ }^{21}$ It is understood that the retinal chromophore is twisted around the C$6 / C-7$ and C-12/C-13 single bonds, and that these twists together with other factors, i.e., the protonated Schiff base and counter anion distance and the electrostatic charge distribution within the binding site, give rise to subtle regulations of absorption maxima of various visual pigments. ${ }^{22}$ The next question to be addressed is the absolute sense of twist around the C-6/C-7 and $\mathrm{C}-12 / \mathrm{C}-13$ single bonds. The twist around the $\mathrm{C}-12 / \mathrm{C}-13$ bond or the twist between planes $\mathrm{B}$ and $C$ (Fig. A) has been solved as follows from the sign of the exciton coupled CD resulting from rhodopsin incorporating 11,12-dihydro-retinal in which the 10-14 moiety is fixed "11-cis", $2 \mathrm{H}$ ret7. 20 

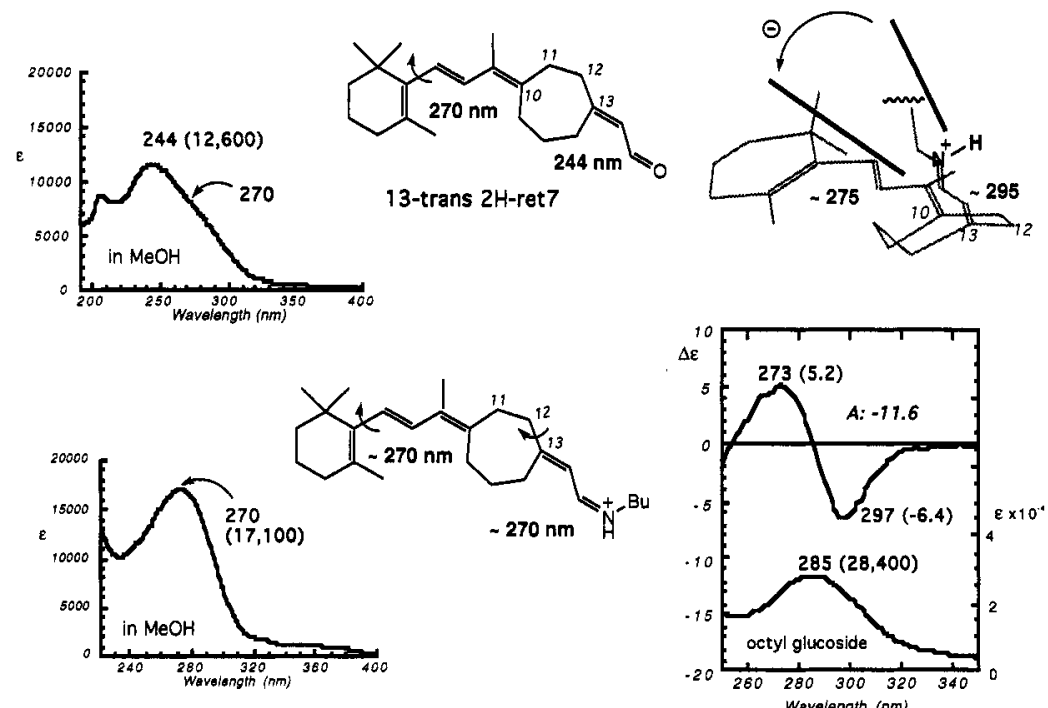

Fig. B

The seven-membered locked dihydro-retinal analog 2H-ret7 (Fig. B) was chosen since similarity in the CD and UV/VIS spectra of the pigment incorporating the ret7, i.e., Rh7, with native rhodopsin indicated that the conformation of the 7 -membered chromophore and the native chromophore were similar. The enal and nonplanar triene moieties in 13-trans- $2 \mathrm{H}$-ret7 absorb at 244 and $270 \mathrm{~nm}$ (shoulder) in methanol, respectively (Fig. B). ${ }^{23}$ In the protonated Schiff base with n-butylamine, the absorption maximum of the enal is shifted to $270 \mathrm{~nm}$; the triene absorption stays the same at $270 \mathrm{~nm}$. When bound to opsin, $2 \mathrm{H}$-ret7 gives a pigment with $\lambda \max$ $285 \mathrm{~nm}$ having a negative split Cotton effect $C D$ at $297 \mathrm{~nm}(\Delta \varepsilon-6.4)$ and $273 \mathrm{~nm}(\Delta \varepsilon+5.2)$, i.e., $A$ value (amplitude) -11.6 (Fig. B). This bisignate nature of the $C D$ is in contrast to native rhodopsin, $\lambda \max 500 \mathrm{~nm}$, with two positive CD Cotton effects at $480 \mathrm{~nm}(\Delta \varepsilon+2.8$, " $\alpha$-band") and $337 \mathrm{~nm}(\Delta \varepsilon+9.8$, " $\beta$-band" $)$. This bisignate $C D$ is interpreted as being due to the coupling between the triene and protonated Schiff base moieties $\left(\mathrm{SBH}^{+}\right)$. Namely, the triene absorption originally at $270 \mathrm{~nm}$ is red-shifted to ca. $275 \mathrm{~nm}$ arising from its more planar shape in the protein, whereas the $270 \mathrm{~nm}$ maximum of the $\mathrm{SBH}^{+}$group is also red-shifted to $295 \mathrm{~nm}$ due to its interaction with the global electrostatic charge within the binding site.
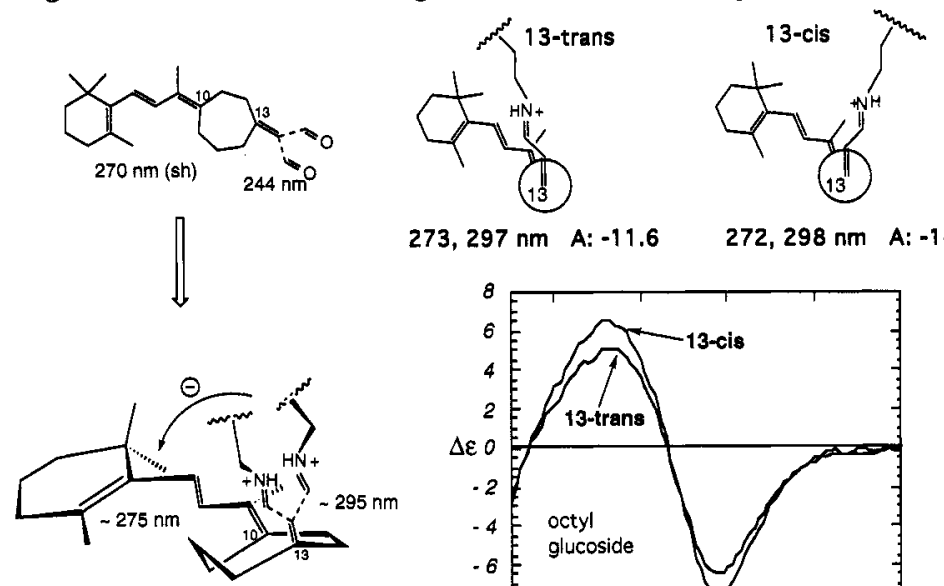

$273,297 \mathrm{~nm} \quad$ A: $-11.6 \quad 272,298 \mathrm{~nm} \quad$ A: -14.2

Fig. C

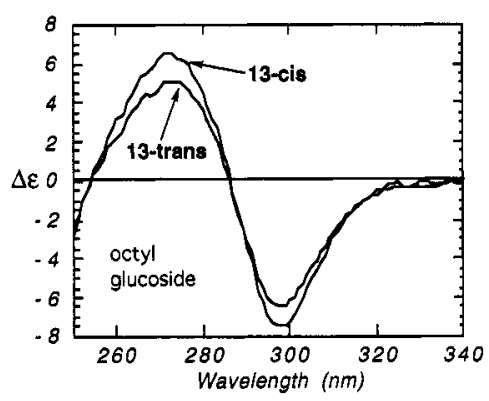

This is supported by the larger $A$ value of -14.2 for the pigment derived from $13-$ cis-2H-ret7 (Fig. C) as compared to the $A$ value of -11.6 for the pigment incorporating 13 -trans-2H-ret7. The 
projection angle of the two interacting chromophores (through C-13 and C-10 axis), is ca. $60^{\circ}$ and ca. $20^{\circ}$, respectively, for 13 -cis and 13-trans isomers; it is known $n^{1,2}$ that the $A$ value of bisignate $\mathrm{CD}$ curves is maximal around $70^{\circ}$. The $A$ value of the pigment derived from the 13-cis isomer is therefore larger.

The pigment analog incorporating 11,12-dihydroretinal without the 7-membered ring absorbs at $279 \mathrm{~nm}(\varepsilon 26,800)$. This pigment also exhibits a negative bisignate $C D, 295 \mathrm{~nm}(\Delta \varepsilon-1.8)$ and $275 \mathrm{~nm}(\Delta \varepsilon+2.7), A$ value -4.5 .20 The amplitude is smaller than those of the $2 \mathrm{H}$-ret7 derived pigments, most probably due to the more flexible nature of the side-chain. Nevertheless, the fact that the $C D$ of 11,12-dihydroretinal rhodopsin is a negative bisignate curve is in agreement with the results of $2 \mathrm{H}$-ret7 rhodopsin, i.e., the triene and $\mathrm{SBH}^{+}$moieties constitute a negative couplet.

The absolute sense of twist between planes $B$ and $C$ is as depicted in Fig. A. The appearance of a clear bisignate $C D$ reflecting the twist of the chromophore present in the rhodopsin binding pocket is a further example of the superb sensitivity and utility of the exciton chirality method.

Acknowledgments. The studies have been supported by NIH GM 34509, 36564 and HL 52852.

\section{REFERENCES}

1. N. Zhao, N. Kumar, K. Neuenschwander, K. Nakanishi, N. Berova, J. Am. Chem. Soc., 117, 7844-7845 (1995).

2. Circular Dichroic Spectroscopy-Exciton Coupling in Organic Stereochemistry, with N. Harada, 1983, pp. 460, University Science Books/Tokyo Kagaku, Dojin.

3. The Exciton Chirality Method, Koji Nakanishi and Nina Berova, in Circular Dichroism, Principles and Applications, ed. K. Nakanishi, N. Berova, R. Woody. VCH Publishers, Inc.: New York, 361-398, (1994).

4. N. Zhao, P. Zhou, N. Berova, K. Nakanishi, Chirality, 7, 636-651 (1995).

5. P. Zhou, N. Zhao, D. N. Rele, N. Berova, K. Nakanishi, J. Am. Chem. Soc., 115, $9313-9314$ (1993).

6. D. Rele, N. Zhao, K. Nakanishi, N. Berova, Tetrahedron, 52, 2759-2776 (1996).

7. N. Zhao, N. Berova, K. Nakanishi, U. J. Jiirgens, Tetrahedron, 52, 2777-2788 (1996).

8. J.-G Dong, W. Bornmann, K. Nakanishi, N. Berova, Phytochemistry, 40, 1821-1824 (1995).

9. a) N. Zhao, L-C. Lo, N. Berova, K. Nakanishi, A. Tymiak, J. Ludens, and G. Haupert, Biochemistry, 34, 98939896 (1995). b) A. Tymiak, J. Norman, M. Bogar, G. DiDonato, H. Lee, W. Parker, L.-C. Lo, N. Berova, K. Nakanishi, E. Haber, G. Haupert, Proc. Natl. Acad. Sci. USA, 90, 8189-8193 (1993). c) J. M. Hamlyn, M. P. Blaustein, S. Bova, D. W. DuCharme, D. W. Harris, F. Mandel, W. R. Mathews, J. H. Ludens, Proc. Natl. Acad. Sci. USA, 88, 6259-6263 (1991).

10. a) R. E. Person, K. Monde, H.-U. Humpf, N. Berova, K. Nakanishi, Chirality, 7, 128-135 (1995). b) J. Su, unpublished.

11. V. Dirsch, J. Frederico, N. Zhao, G. Cai, Y. Chen, S. Vunnam, J. Odingo, H. Pu, K. Nakanishi, N. Berova, D. Liotta, A. Bielawska, Y. Hannun, Tetrahedron Lett., 36, 4959-4962 (1995).

12. A. Kawamura, N. Berova, V. Dirsch, A. Mangoni, K. Nakanishi, G. Schwarz, A. Bielawska., Y. Yannun, I. Kitagawa, Bioorganic and Biomedical Chemistry, 4, 1035-1043 (1996).

13. S. Matile, N. Berova, K. Nakanishi, S. Novkova, 1. Philipova, and B. Blagoev, J. Am. Chem. Soc., 117, 70217022 (1995).

14. S. Matile, N. Berova, K. Nakanishi, J. Fleischhauer, R. Woody, J. Am. Chem. Soc., 118, 5198-5206 (1996).

15. S. Matile, N. Berova, K. Nakanishi, Enantiomer. 1, 1-12 (1996); B. Rickman, unpublished.

16. S. Matile, N. Berova, K. Nakanishi, Chem. \& Biol., 3, 379-392 (1996).

17. S. Matile, K. Nakanishi, Angew. Chem., 35, 757-759 (1996).

18. Y. Chen, P. Zhou, N. Berova, H. Zhang, K. Nakanishi, J. Am. Chem. Soc., 116, 2683-2684 (1994).

19. H. Zhang, K. Lerro, T. Yamamoto, T. Lien, L. Sastry, M. Gawinowicz, K. Nakanishi, J. Am. Chem. Soc., 116, 10165-10173, (1994); K. Nakanishi, H. Zhang, K. Lerro, S. Takekurna, T. Yarnamoto, T. Lien, L. Sastry, D. Baek, C. Moquin-Pattey, M. Boehm, F. Derguini, M. Gawinowicz, Biophysical Chemistry, 56, 13-22 (1995).

20. Q. Tan, J. Lou, B. Borhan, E. Karnaukhova, N. Berova, K. Nakanishi, to be published.

21. See also T. A., Nakayama and H. G. Khorana, J. Biol. Chem. 265, 15762 (1990).

22. K. Nakanishi, Pure \& Applied Chemistry, 63, 161-170 (1991).

23. H. Akita, S. Tanis, M. Adams, V. Balogh-Nair, K. Nakanishi, J. Am. Chem. Soc., 102, 6370 (1980). 